\title{
Antimicrobial activity and the mechanism of silver nanoparticle thermosensitive gel
}

\author{
This article was published in the following Dove Press journal: \\ International Journal of Nanomedicine \\ I4 November 201 I \\ Number of times this article has been viewed
}

\section{Meiwan Chen ${ }^{1,2, \ddagger}$ \\ Zhiwen Yangl,f \\ Hongmei Wu' \\ Xin Pan' \\ Xiaobao $\mathrm{Xie}^{3}$ \\ Chuanbin $\mathrm{Wu}^{\mathrm{I}}$}

'Research and Development Center of Pharmaceutical Engineering, School of Pharmaceutical Sciences, Sun Yatsen University, Guangzhou, China; ${ }^{2}$ State Key Laboratory of Quality Research in Chinese Medicine, Institute of Chinese Medical Sciences, University of Macau, Macau, China; ${ }^{3}$ Guangdong Provincial Key Laboratory of Microbial Culture Collection and Application, Guangzhou, China

¥These authors contributed equally to this work
Correspondence: Chuanbin Wu School of Pharmaceutical Sciences, Sun Yat-sen University, Guangzhou 510006, China

Tel +86 2039943II 7

Fax +862039943120

Email cbwu2000@yahoo.com
Purpose: The purpose of the present study was to elucidate the antimicrobial activity and mechanism of silver nanoparticles incorporated into thermosensitive gel (S-T-Gel) on Staphylococcus aureus, Escherichia coli, and Pseudomonas aeruginosa.

Patients and methods: This study investigated the growth, permeability, and morphology of Staphylococcus aureus, Escherichia coli, and Pseudomonas aeruginosa cells in order to observe the action of S-T-Gel on the membrane structure of these three bacteria. The cell morphology of normal and treated bacteria cells was assessed by transmission electron microscopy (TEM), and the effects of S-T-Gel on genome DNA of bacterial cells were evaluated by agarose gel electrophoresis.

Results: S-T-Gel showed promising activity against Staphylococcus aureus and moderate activity against Escherichia coli and Pseudomonas aeruginosa. The observation with TEM suggested that S-T-Gel may destroy the structure of bacterial cell membranes in order to enter the bacterial cell. S-T-Gel then condensed DNA and combined and coagulated with the cytoplasm of the damaged bacteria, resulting in the leakage of the cytoplasmic component and the eventual death of these three bacteria. In addition, the analysis of agarose gel electrophoresis demonstrated that S-T-Gel could increase the decomposability of genome DNA.

Conclusion: These results about promising antimicrobial activity and mechanism of S-T-Gel may be useful for further research and development in in-vivo studies.

Keywords: molecule mechanism, bacterial cells, S-T-Gel

\section{Introduction}

Silver nanoparticles have been developed as a potent antibacterial, antifungal, antiviral, and anti-inflammatory agent. ${ }^{1-3}$ People have utilized silver nanoparticles since ancient times for medicinal purposes as well as for their attractive physical, chemical, and biological properties. ${ }^{4,5}$ Compared with other metals, silver nanoparticles show higher toxicity to microorganisms while exhibiting lower toxicity to mammalian cells. ${ }^{6}$ To date, the most promising applications have been shown in the medical or pharmacological fields, such as biosensors ${ }^{7}$ and wound treatment for infection. ${ }^{8,9}$ Silver nanoparticles are available as an antimicrobial gel formulation for conventional topical antimicrobial agents, especially for burn treatment. ${ }^{10}$ However, conventional gels and ointments have certain disadvantages, such as the need for a special application syringe to deliver the gel, which may soften or liquidate unpredictably, and move away from the treatment site rapidly. The gel would not reside on the required region for a long time, resulting in poor patient compliance and unpredictable therapeutic effects.

In the past decades, considerable attention has been focused on the development of novel and controlled-release drug-delivery systems to provide a long-term therapeutic 
concentration of drugs following a single dose. ${ }^{11}$ Many controlled-release drug-delivery systems are based on hydrogels, especially environment-sensitive hydrogels such as thermosensitive hydrogels. Therefore, various synthetic thermosensitive polymers have been extensively studied in pharmaceutical and biomedical applications. Pluronics is a class of thermosensitive polymers that have been widely utilized as drug carriers, since they exhibit unique sol-gel transition behaviors in response to temperature in aqueous solution. ${ }^{12}$ Pluronic F127 and F68 are nonionic surfactant/ difunctional block copolymers that terminate in primary hydroxyl groups, are $100 \%$ active, and are relatively nontoxic. ${ }^{13}$ Making a thermosensitive gel by Pluronics was an appealing dosage form that had lots of advantages over conventional methods, such as it may increase residence time at the absorption site, improve contact between the delivery system and the absorption site, and provide localization to specified oral mucosa regions to enhance bioavailability. The authors' previous studies showed that silver nanoparticles incorporated into Pluronic F127 and F68 to make thermosensitive gel containing silver nanoparticles (S-T-Gel) might provide a long-term effective concentration of the drug following a single dose, thereby leading to an efficient therapeutic result for bacterial infection. ${ }^{14}$

Previous studies also showed that there are several mechanisms about the bactericidal effect of silver nanoparticles. Silver nanoparticles may attach to the surface of the cell membrane, interrupting permeability and metabolic pathways of the cell. ${ }^{15}$ Silver nanoparticles not only interact with the surface of the membrane, but can also penetrate into the bacterial cell membrane. ${ }^{3}$ In addition, silver nanoparticles can bind to the DNA inside the bacterial cells, preventing its replication or interaction with the bacterial ribosome. ${ }^{16,17}$ It has been discovered that silver nanoparticles can damage the structure of the bacterial cell membrane and reduce the activity of some membranous enzymes, which cause $E$. coli bacteria to die eventually. ${ }^{18}$ However, no research has been reported in the literature to investigate the antimicrobial activity and mechanism of silver nanoparticles when they are incorporated into Pluronic F127 and F68. Therefore, in the present study, S-TGel was prepared by Pluronic F127 and F68 and its antimicrobial activity and mechanism also have been investigated. To investigate whether the thermosensitive gel will affect the antimicrobial activity and mechanism of silver nanoparticles, antimicrobial activity and mechanism of S-T-Gel were evaluated. Therefore, the growth, permeability, and morphology of the bacterial cells following treatment with S-T-Gel were investigated in this study.

\section{Materials and methods Materials}

Silver nanoparticles were purchased from Shanghai Tinaph Nano-Tech Co, Ltd (Shanghai, China), glycerol from Guangzhou-hung Instrument Co, Ltd (Guangzhou, China), and ethylparaben from the Development Center of Tianjin Kemiou Chemreagent (Tianjin, China). Pluronic ${ }^{\circledR}$ F127 and F68 used for thermosensitive gel preparation were obtained from BASF (Ludwigshafen, Germany). Double-distilled and deionized water was used throughout the experiment, and all other materials used were of analytical or pharmaceutical grade.

\section{Microorganisms and growth conditions}

Staphylococcus aureus (ATCC 25923), Escherichia coli (ATCC 25922), and Pseudomonas aeruginosa (ATCC 27853) were purchased from American Type Culture Collection (ATCC, Manassas, VA). Bacterial strains were cultivated on Mueller-Hinton medium (pH $7.3 \pm 0.2$; Guangdong Huankai Microbial Science and Technology Co Ltd, Guangzhou, China) at $37^{\circ} \mathrm{C}$ with shaking at $150 \mathrm{rpm}$. Cell suspensions were diluted with a sterile saline solution to obtain a final concentration of $10^{7} \mathrm{CFU} / \mathrm{mL}$ by comparison with a 0.5 McFarland turbidity standard. Growth curves of $S$. aureus, E. coli, and $P$. aeruginosa, exposed and unexposed to S-T-Gel, were determined based on the absorbance value at optical density measured at a wavelength of $600 \mathrm{~nm}\left(\mathrm{OD}_{600}\right)$.

\section{Preparation of S-T-Gel formulation}

Glycerol and ethylparaben were dissolved in double-distilled and deionized water to make a working concentration of $1.0 \%$ and $0.1 \%$, respectively. Pluronic F127 (12.0 wt \%) and F68 (6.0 wt $\%)$ were prepared in aqueous glycerol and ethylparaben according to the cold method described by El-Kamel. ${ }^{19}$ Silver nanoparticles were incorporated into this gel (S-T-Gel) to achieve a final silver concentration of $350 \mathrm{mg} / \mathrm{L}$. All formulations were allowed to equilibrate to $25^{\circ} \mathrm{C}$ for 24 hours before performing experimental studies.

\section{Growth curve of S-T-Gel to bacteria}

To determine the growth curves of S-T-Gel to $S$. aureus, E. coli, and P. aeruginosa, $20 \mathrm{~mL}$ Luria-Bertani (LB) broth medium, S-T-Gel, and bacteria were added separately to five conical flasks $(50 \mathrm{~mL})$ in order to obtain the final S-T-Gel concentrations of $0.0,0.5,1.0,2.0,5.0$, and $10.0 \mathrm{mg} / \mathrm{L}$ and bacterial cell concentration of $10^{6} \mathrm{CFU} / \mathrm{mL}$. The six conical flasks were incubated in an upright position at $37^{\circ} \mathrm{C} \pm 2{ }^{\circ} \mathrm{C}$ and shaken at $150 \mathrm{rpm}$ under $5 \% \mathrm{CO}_{2}$. Growth rates and bacterial concentrations were determined by measuring 
$\mathrm{OD}_{600}{ }^{20}$ through a series of time intervals $(2,4,6,8,10,12$, 24, 36, and 48 hours).

\section{Observation of interaction between S-T-Gel and bacteria}

The interaction between $S$. aureus, E. coli, and P. aeruginosa and S-T-Gel was examined by transmission electron microscopy (TEM) (Hitachi H-7650; Pleasanton, CA). LB broth medium $(10 \mathrm{~mL}), \mathrm{S}-\mathrm{T}-\mathrm{Gel}$, and bacteria were added to a $20 \mathrm{~mL}$ test tube to obtain the final concentrations of $10 \mathrm{mg} / \mathrm{L}$ $\mathrm{S}-\mathrm{T}-\mathrm{Gel}$ and $10^{6} \mathrm{CFU} / \mathrm{mL}$ bacteria cells. Control group was generated without S-T-Gel. The test tubes were incubated at $37^{\circ} \mathrm{C} \pm 2^{\circ} \mathrm{C}$ and shaken at $150 \mathrm{rpm}$ under $5 \% \mathrm{CO}_{2}$ for 12 hours, after which the cultures were centrifuged and the pellets harvested for morphology and structure analysis by TEM according to the one described by Li et al. ${ }^{18}$

\section{Assessment of the effect of S-T-Gel on the DNA of bacteria}

To determine whether the S-T-Gel has an influence on the DNA of damaged bacteria, the amount of DNA from normal bacteria cells and bacteria cells treated by S-T-Gel were evaluated by agarose gel electrophoresis. The reaction mixture containing $0.5 \mathrm{~mL}$ LB broth medium, S-T-Gel, and bacteria in $20 \mathrm{mM}$ potassium phosphate buffer $(\mathrm{pH}$ 7.4) was preincubated for 30 minutes at $37^{\circ} \mathrm{C}$.

\section{Statistical analysis}

Means and standard deviations were obtained from three experiments and were analyzed by one-way analysis of variance followed by Dunnett's multiple-comparison test. Statistically significant differences were identified when $P<0.05$.

\section{Results and discussion Growth curve of S-T-Gel to bacteria}

Growth curves of $S$. aureus, E. coli, and P. aeruginosa treated with different concentrations of S-T-Gel $(0.0,0.5$, $1.0,2.0,5.0$, and $10.0 \mathrm{mg} / \mathrm{L}$ ) included four phases: lag phase, exponential phase, stabilization phase, and decline phase, as shown in Figure 1. Stabilization phase and decline phase could not be separated because we only used the value of the $\mathrm{OD}_{600}$ value to assay the total amount of bacteria living and dead. Compared with the control group, S-T-Gel can extend the lag phase of $S$. aureus, E. coli, and $P$. aeruginosa. The higher the concentration of S-T-Gel, the longer the lag phase of $S$. aureus, E. coli, and P. aeruginosa. The effects were shown to be concentration-dependent;
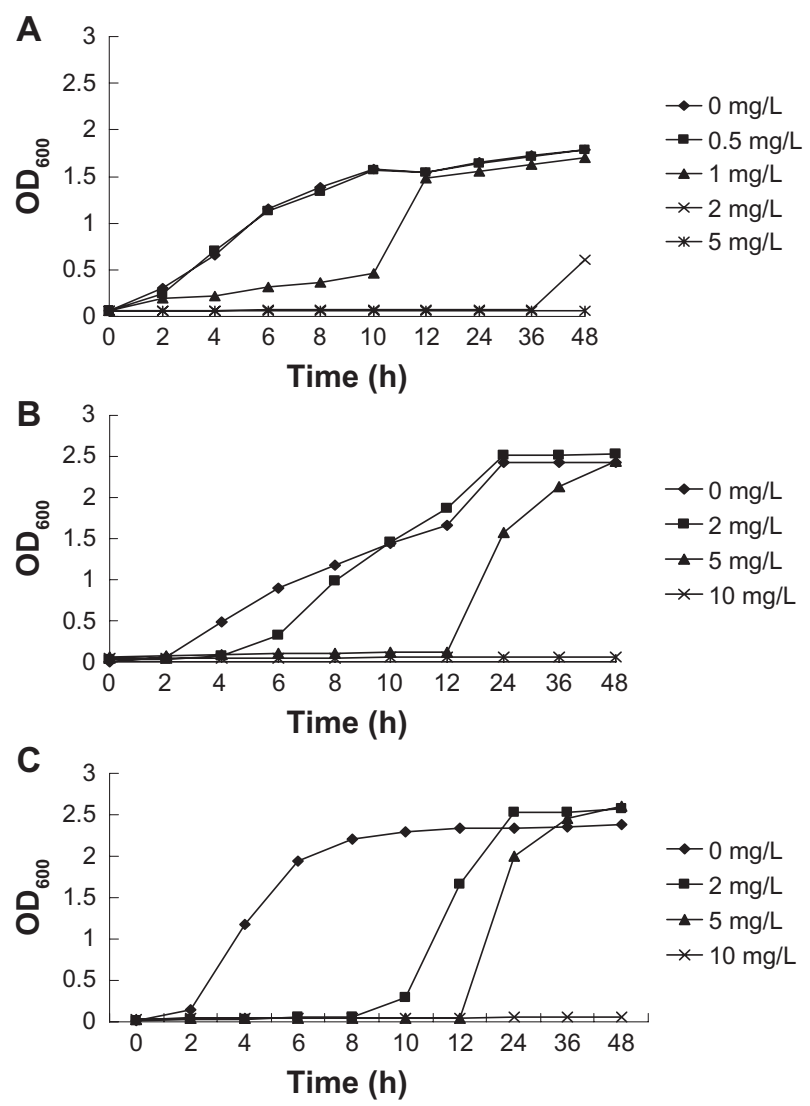

Figure I Growth curves of different concentrations of S-T-Gel with (A) Staphylococcus aureus, (B) Escherichia coli, and (C) Pseudomonas aeruginosa.

Abbreviation: S-T-Gel, silver nanoparticles incorporated into thermosensitive gel.

when the concentration of S-T-Gel was $5 \mathrm{mg} / \mathrm{L}$, the growth of $10^{7} \mathrm{CFU} / \mathrm{mL}$ S. aureus was completely inhibited by 48 hours. In addition, no growth of $E$. coli and $P$. aeruginosa could be detected when the concentration of S-T-Gel reached $10 \mathrm{mg} / \mathrm{L}$. S-T-Gel showed promising activity against $S$. aureus and moderate activity against $E$. coli and $P$. aeruginosa.

\section{Observation of interaction between S-T-Gel and bacteria}

Morphology and structure of normal S. aureus, E. coli, and $P$. aeruginosa cells, and $S$. aureus, E. coli, and P. aeruginosa cells treated by S-T-Gel for 12 hours were observed using TEM (Figure 2). The cell structures are different between Gram-positive bacteria ( $S$. aureus) and Gram-negative bacteria (E. coli and P. aeruginosa). The cell wall of Gram-positive bacteria is thick and made of peptidoglycan and teichoic acid. The composition of peptide chain between peptidoglycan was cross-linked by five glycines. However, the cell wall of Gram-negative bacteria is a multilayer structure, which from the inside to outside are as follows: a thin peptidoglycan, lipoprotein/cortical, phospholipid, and lipopolysaccharide. 
A
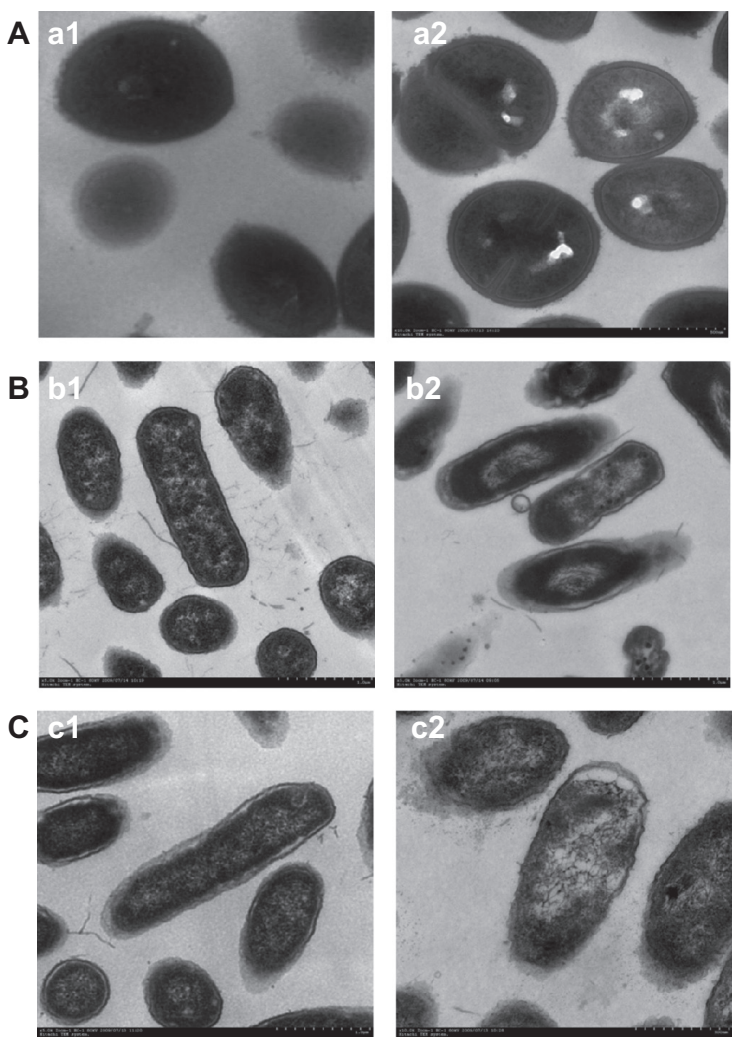

Figure 2 Morphology and structure of bacterial cells under transmission electron microscopy. (A) al, normal Staphylococcus aureus cells; a2, S. aureus cells treated by S-T-Gel. (B) bl, normal Escherichia coli cells; b2, E. coli cells treated by S-T-Gel. (C) cl, normal Pseudomonas aeruginosa cells; c2, P. aeruginosa cells treated by S-T-Gel. Abbreviation: S-T-Gel, silver nanoparticles incorporated into thermosensitive gel.

The peptidoglycan structure between Gram-negative bacteria and Gram-positive bacteria is different, as peptidoglycan was directly cross-linked together in Gram-negative bacteria. Sensitivity of Gram-positive bacteria and Gram-negative bacteria to different kinds of antibiotics is different, because the cell wall structure of them is different.

As shown in Figure 2A, the cell membrane of S. aureus cells treated by S-T-Gel was lighter. The cell membrane and cell wall is easily distinguished. There is the same change between E. coli and P. aeruginosa as they are negative bacteria. In the control group, the electron density is evenly distributed in E. coli and P. aeruginosa cells, which is a typical morphological characteristic in these normal cells. The lighter material in the cell is DNA molecules, which were randomly distributed in the nuclear area of $E$. coli and $P$. aeruginosa cells. Within the treatment group, there are large changes in internal structure of E. coli and P. aeruginosa cells. In Figure 2B and C, there are obvious bright electron areas in the center of bacteria cells. In the bright electron areas, there are some materials which concentrated very closely and showed a linear state. The bright region is also surrounded by many electron dense granules. The reason may be S-T-Gel caused the separation of the cell wall and cell membrane, resulting in the condensed and tense state of DNA. Many electron dense granules may be S-T-Gel, while in the bright region there is no electron dense granules; this is due to the bright region keeping S-T-Gel away.

\section{Assessment of the effect of S-T-Gel on the DNA of bacteria}

To confirm whether S-T-Gel can increase the decomposability of genome DNA, the analysis of agar gel electrophoresis was performed. Agar gel electrophoresis images as shown in Figure 3 revealed that the bands about genome DNA of normal $S$. aureus, E. coli, and P. aeruginosa respectively are lighter than the ones about genome DNA of $S$. aureus, E. coli, and P. aeruginosa cells treated with $10 \mu \mathrm{g} / \mathrm{mL}$ S-T-Gel respectively. These results confirmed that S-T-Gel can reduce the amount of genome DNA of S. aureus, E. coli, and P. aeruginosa cells.

Although some literature reports bacterial cells with negative charge can interact with silver nanoparticles with positive charge due to electrostatic interaction, there are no complete reports about how silver nanoparticles affect the process of damaging bacteria and the impact to bacterial DNA. In a previous study, Guangdong Provincial Key Laboratory of Microbial Culture Collection and Application, China, carried out research on the "study of antimicrobial activity and mechanism of silver" and reported the changes of $E$. coli as being progressive. ${ }^{18}$ Silver nanoparticles lead to the formation of "pits" in the cell walls of the bacteria and can enter into the periplasm through the pits and destroy the cell membrane. Silver nanoparticles not only condense DNA, but also combine and coagulate with the cytoplasm of damaged bacteria, which results in the leakage of the cytoplasmic component. Silver nanoparticles may cause the condensing of DNA, resulting in a loss of replication and

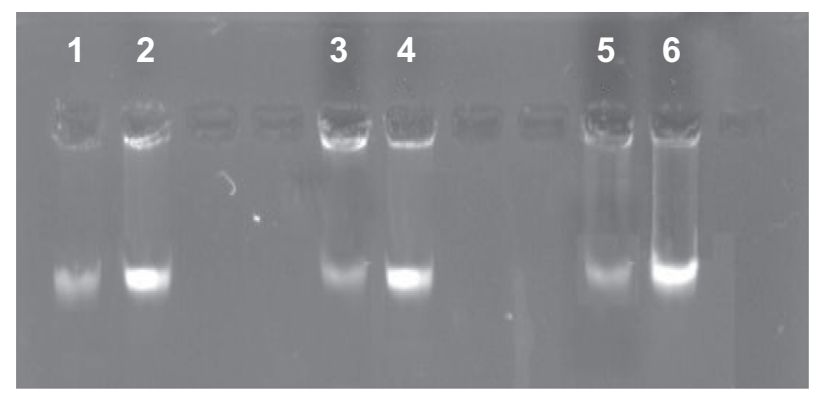

Figure 3 Effect of S-T-Gel on genome DNA of bacterial cells.

Note: 2, 4, and 6 denote genome DNA of normal Staphylococcus aureus, Escherichia coli, and Pseudomonas aeruginosa cells respectively. I, 3, and 5 denote genome DNA of S. aureus, E. coli, and $P$. aeruginosa cells treated with $10 \mu \mathrm{g} / \mathrm{mL}$ S-T-Gel respectively. Abbreviations: DNA, deoxyribonucleic acid; S-T-Gel, silver nanoparticles incorporated into thermosensitive gel. 
degradation of DNA, thereby inhibiting bacterial growth. There is condensed DNA in the center of the bright electron areas which appears in the middle of bacteria cells. In the present study, the analysis of agar gel electrophoresis was performed to see the effects of S-T-Gel on the genome DNA of bacterial cells. This is the first time to report the mechanism of silver nanoparticles incorporated into thermosensitive gel on S. aureus, E. coli, and P. aeruginosa. There have been no previous reports on the subject. The results showed that S-T-Gel can also change the DNA of bacterial cells and inhibit the growth of $S$. aureus, E. coli, and P. aeruginosa cells.

\section{Conclusion}

Silver nanoparticles incorporated into thermosensitive gel (S-T-Gel) have great potential application for bacterial infection. This work described the preparation of S-T-Gel and investigated its antimicrobial activity and the mechanism. It demonstrated that S-T-Gel showed promising activity against $S$. aureus and moderate activity against $E$. coli and P. aeruginosa. S-T-Gel may destroy the structure of bacterial cell membranes to enter the bacterial cell, then condense DNA and combine and coagulate with the cytoplasm of the damaged bacteria, resulting in the leakage of the cytoplasmic component and the eventual death of bacteria. In addition, S-T-Gel can increase the decomposability of genome DNA. The antimicrobial activity and the mechanism of S-T-Gel developed in this work possesses the potential to serve as a platform for developing silver nanoparticles into a novel dosage form. To study and develop S-T-Gel, characteristics and quality control should be strengthened in future studies.

\section{Acknowledgments}

The work was supported by National Science and Technology Foundation of China "The Creation for Significant New Drugs" (2009ZX09501-023), the Science and Technology Planning Project of Guangdong Province, China (Project No. 2007B03070400), and the University of Macau research fund (UL016A/09-Y2/CMS/WYT01/ICMS).

\section{Disclosure}

The authors declare that they have no competing interests.

International Journal of Nanomedicine

\section{Publish your work in this journal}

The International Journal of Nanomedicine is an international, peerreviewed journal focusing on the application of nanotechnology in diagnostics, therapeutics, and drug delivery systems throughout the biomedical field. This journal is indexed on PubMed Central, MedLine, CAS, SciSearch ${ }^{\circledR}$, Current Contents ${ }^{\circledR} /$ Clinical Medicine,

\section{References}

1. Vaidyanathan R, Kalishwaralal K, Gopalram S, Gurunathan S. Nanosilver - the burgeoning therapeutic molecule and its green synthesis. Biotechnol Adv. 2009;27(6):924-937.

2. Panacek A, Kolar M, Vecerova R, et al. Antifungal activity of silver nanoparticles against Candida spp. Biomaterials. 2009;30(31):6333-6340.

3. Elechiguerra JL, Burt JL, Morones JR, et al. Interaction of silver nanoparticles with HIV-1. J Nanobiotechnology. June 29, 2005;3:6.

4. Elechiguerra JL, Burt JL, Morones JR, et al. Interaction of silver nanoparticles with HIV-1. J Nanobiotechnol. 2005;3(6):10.

5. Pal S, Tak YK, Song JM. Does the antibacterial activity of silver nanoparticles depend on the shape of the nanoparticle? A study of the Gram-negative bacterium Escherichia coli. Appl Environ Microbiol. 2007;73(6):1712-1720.

6. Zhao G, Stevens SE Jr. Multiple parameters for the comprehensive evaluation of the susceptibility of Escherichia coli to the silver ion. Biometals. 1998;11(1):27-32.

7. Sun H, Choy TS, Zhu DR, Yam WC, Fung YS. Nano-silver-modified $\mathrm{PQC} / \mathrm{DNA}$ biosensor for detecting $E$. coli in environmental water Biosens Bioelectron. 2009;24(5):1405-1410.

8. Lu S, Gao W, Gu HY. Construction, application and biosafety of silver nanocrystalline chitosan wound dressing. Burns. 2008;34(5):623-628.

9. Muangman P, Chuntrasakul C, Silthram S, et al. Comparison of efficacy of $1 \%$ silver sulfadiazine and Acticoat for treatment of partial-thickness burn wounds. J Med Assoc Thai. 2006;89(7):953-958.

10. Jain J, Arora S, Rajwade JM, Omray P, Khandelwal S, Paknikar KM. Silver nanoparticles in therapeutics: development of an antimicrobia gel formulation for topical use. Mol Pharm. 2009;6(5):1388-1401.

11. Mandal TK. Swelling-controlled release system for the vaginal delivery of miconazole. Eur J Pharm Biopharm. 2000;50(3):337-343.

12. Chung HJ, Lee Y, Park TG. Thermo-sensitive and biodegradable hydrogels based on stereocomplexed Pluronic multi-block copolymers for controlled protein delivery. J Control Release. 2008;127:22-30.

13. Zhao X-Y, Xu J, Zheng L-Q, Li X-W. Preparation of temperaturesensitive microemulsion-based gels formed from a triblock copolymer. Coll Surf A Physicochem Eng Aspects. 2007;307:100-107.

14. Chen M, Pan X, Wu H, et al. Preparation and anti-bacterial properties of a temperature-sensitive gel containing silver nanoparticles. Pharmazie. 2011;66(4):272-277.

15. Sharma HS, Hussain S, Schlager J, Ali SF, Sharma A. Influence of nanoparticles on blood-brain barrier permeability and brain edema formation in rats. Acta Neurochir Suppl. 2010;106:359-364.

16. Yang W, Shen C, Ji Q, et al. Food storage material silver nanoparticles interfere with DNA replication fidelity and bind with DNA Nanotechnology. 2009;20(8):085102.

17. Lu L, Sun RW, Chen R, et al. Silver nanoparticles inhibit hepatitis B virus replication. Antivir Ther. 2008;13(2):253-262.

18. Li WR, Xie XB, Shi QS, Zeng HY, Ou-Yang YS, Chen YB. Antibacterial activity and mechanism of silver nanoparticles on Escherichia coli. Appl Microbiol Biotechnol. 2010;85(4):1115-1122.

19. El-Kamel AH. In vitro and in vivo evaluation of Pluronic F127based ocular delivery system for timolol maleate. Int J Pharm. 2002;241(1):47-55.

20. Podsiadlo P, Paternel S, Rouillard JM, et al. Layer-by-layer assembly of nacre-like nanostructured composites with antimicrobial properties. Langmuir. 2005;21(25):11915-11921.

\section{Dovepress}

Journal Citation Reports/Science Edition, EMBase, Scopus and the Elsevier Bibliographic databases. The manuscript management system is completely online and includes a very quick and fair peer-review system, which is all easy to use. Visit http://www.dovepress.com/ testimonials.php to read real quotes from published authors. 